\title{
Generation, fractionation, and characterization of iron-chelating protein hydrolysate from palm kernel cake proteins
}

\begin{abstract}
Palm kernel cake protein was hydrolyzed with different proteases namely papain, bromelain, subtilisin, flavourzyme, trypsin, chymotrypsin, and pepsin to generate different protein hydrolysates. Peptide content and iron-chelating activity of each hydrolysate were evaluated using O-phthaldialdehyde-based spectrophotometric method and ferrozine-based colorimetric assay, respectively. The results revealed a positive correlation between peptide contents and iron-chelating activities of the protein hydrolysates. Protein hydrolysate generated by papain exhibited the highest peptide content of $10.5 \mathrm{mM}$ and highest iron-chelating activity of $64.8 \%$ compared with the other hydrolysates. Profiling of the papain-generated hydrolysate by reverse phase high performance liquid chromatography fractionation indicated a direct association between peptide content and iron-chelating activity in most of the fractions. Further fractionation using isoelectric focusing also revealed that protein hydrolysate with basic and neutral isoelectric point (pI) had the highest iron-chelating activity, although a few fractions in the acidic range also exhibited good metal chelating potential. After identification and synthesis of papain-generated peptides, GGIF and YLLLK showed among the highest iron-chelating activities of $56 \%$ and $53 \%$, whereas their IC50 were 1.4 and 0.2 عM, respectively.
\end{abstract}

Keyword: Chelating activity; Palm kernel cake; Peptide; Protein hydrolysate 